\title{
Spectral constraints on unidentified EGRET gamma-ray sources from COMPTEL MeV observations
}

\author{
S. Zhang ${ }^{1,2}$, W. Collmar ${ }^{2}$, W. Hermsen ${ }^{3,4}$, and V. Schönfelder ${ }^{2}$ \\ ${ }^{1}$ Laboratory of Cosmic Ray and High Energy Astrophysics, Institute of High Energy Physics, PO Box 918-3, Beijing 100039, \\ PR China \\ 2 Max-Planck-Institut für extraterrestrische Physik, PO Box 1603, 85740 Garching, Germany \\ 3 SRON National Institute for Space Research, Sorbonnelaan 2, 3584 CA Utrecht, The Netherlands \\ 4 Astronomical Institute “Anton Pannekoek”, University of Amsterdam, Kruislaan 403, 1098 SJ Amsterdam, The Netherlands
}

Received 13 November 2003 / Accepted 31 March 2004

\begin{abstract}
We investigated the $\mathrm{MeV}$ properties of 173 unidentified or tentatively identified EGRET sources listed in the third EGRET catalogue by analyzing the simultaneously collected COMPTEL MeV data for each individual source. The sources can be divided into 4 groups. In this paper we focus on one of these, a group of 22 EGRET sources for which we can provide additional constraining information: their spectral extrapolations from the energy range above $100 \mathrm{MeV}$ towards lower energies overshoot the fluxes or upper limits derived simultaneously at $\mathrm{MeV}$ energies. This means that for these sources a spectral turnover/break between $1 \mathrm{MeV}$ and $100 \mathrm{MeV}$ is required. At least two of these sources, but most likely the majority of this sample, have the maxima of their gamma-ray luminosities in this energy band. The sources have rather soft EGRET spectra (average photon index $=2.72_{-0.11}^{+0.08}$ ), and seem to spatially cluster in the inner Galaxy. Variability analyses revealed 11 out of the 22 sources to be significantly variable. Object classes proposed as possible counterparts for the unidentified EGRET sources are discussed in the light of these additional constraints.
\end{abstract}

Key words. $\gamma$-rays: observations

\section{Introduction}

One of the biggest mysteries left by the Compton Gamma-Ray Observatory (CGRO, 1991-2000) is that a large number of $\gamma$ ray sources detected by the different CGRO experiments, in particular EGRET, still remain unidentfied. The EGRET experiment measured $\gamma$-rays above $30 \mathrm{MeV}$, most sensitively above $100 \mathrm{MeV}$. Out of the 271 sources listed in the third EGRET catalogue (Hartman et al. 1999), 170 are unidentified and 27 are only tentatively identified. Several classes of objects have been proposed as possible counterparts for those unidentified EGRET sources. Sources located at high galactic latitudes and being time variable are believed to be active galactic nuclei (AGN), in particular blazars. Sources, located at lower galactic latitudes, being steady and having low $\gamma$-ray fluxes, are found to coincide spatially with the Gould Belt (Gehrels et al. 2000). Some other low-latitude sources show positional correlations with supernova remnants (SNRs) and OB associations (e.g., Romero et al. 1999). Steady sources with hard $\gamma$-ray spectra seem to be good candidates for young $\gamma$-ray pulsars with ages of less than $10^{6}$ years (Zhang et al. 2000a). Several sources (mainly located $|b|<10^{\circ}$ ) might indicate a new class of $\gamma$-ray

Send offprint requests to: W. Collmar, e-mail: wec@mpe .mpg. de emitting objects (Torres et al. 2001), because they do not coincide with any potential counterpart objects.

The COMPTEL experiment aboard CGRO is sensitive to $\gamma$-ray photons between 0.75 and $30 \mathrm{MeV}$, thereby covering the softer $\gamma$-ray band adjacent to the EGRET one. Apart from transient $\gamma$-ray bursts, unidentified $\gamma$-ray sources and AGN are the majority of the COMPTEL source detections. The first COMPTEL catalogue (Schönfelder et al. 2000) lists 10 AGN and 9 unidentified $\gamma$-ray sources; the sum of the rest (radio pulsars, stellar black-hole candidates, SNRs, and $\gamma$-ray line sources) is about 12. Since COMPTEL and EGRET were mounted parallel on CGRO and both had a large field of view (the COMPTEL one being larger than the EGRET one), COMPTEL and EGRET observed simultaneously the same sky region.

To gain further knowledge on the unidentified EGRET sources, and to probe their nature, we analyzed the contemporaneous COMPTEL data on the unidentified EGRET sources to supplement the EGRET results. In this paper, we report the discovery of a subgroup of the unidentified EGRET sources whose $\gamma$-ray spectra are constrained by the $\mathrm{MeV}$ data: their spectral energy distributions have at least an $\mathrm{MeV}$ break but most likely an $\mathrm{MeV}$ peak. The paper 
is organized as follows: in Sect. 2 we briefly describe the COMPTEL instrument, the applied data analysis methods and the observational concept of CGRO, in Sect. 3 we present the analysis results and discuss them in Sect. 4. In Sect. 5, we finally present the conclusions.

\section{Instrument, data analysis and CGRO observations}

The Compton telescope COMPTEL (0.75-30 MeV) had an energy-dependent energy and angular resolution of 5\%-8\% $(F W H M)$ and $1.7^{\circ}-4.4^{\circ}(F W H M)$, respectively. Its field of view is circular and covers $\sim 1$ steradian. Imaging in its large field of view is possible with a location accuracy (flux dependent) of the order of $1^{\circ}-3^{\circ}$. For details on the experiment see Schönfelder et al. (1993).

Skymaps and source parameters, like detection significances, fluxes, and flux errors, can be obtained via the maximum likelihood method, which is implemented in the standard COMPTEL data analysis package. The detection significance is derived from the quantity $-2 \ln \lambda$, where $\lambda$ is the ratio of the likelihood $L_{0}$ (background) to the likelihood $L_{1}$ (source + background). The quantity $-2 \ln \lambda$ has a $\chi_{1}^{2}$ distribution, if only the flux at a given source position is estimated (de Boer et al. 1992). The detection significance can be conservatively calculated by the ratio of flux to flux error. This approach is adopted in this paper for estimating the source detection significances.

In fitting the fluxes of the relevant EGRET sources, nearby prominent COMPTEL sources are taken into account by fitting simultaneously their fluxes. An estimate for the instrumental background of COMPTEL is derived by using the standard filter technique in the COMPTEL data space (Bloemen et al. 1994). The celestial background components, galactic and extra-galactic diffuse $\gamma$-ray radiation, also have been taken into account by model fitting. In the presented analyses we applied instrumental point spread functions assuming an $E^{-2}$ powerlaw shape for the source spectra.

CGRO observations were organized in so-called "Mission Phases" and "Viewing Periods (VPs)". A "Mission Phase" covers typically 1 year of data and contains many VPs, which typically last for 1 to 2 weeks each. For each EGRET source of interest we analyzed the simultaneously collected COMPTEL data. To allow combining of COMPTEL and EGRET results, we analyzed the $\mathrm{MeV}$ data for periods for which the EGRET spectral index was estimated. Many EGRET source results are published in the sum of the CGRO Mission Phases 1 to 4, noted as "P1234" in the third EGRET catalogue. For this time period we generated COMPTEL all-sky data, and derived the flux results by fitting sources at the relevant positions. As noted above, the fit was performed by including 1) models for the cosmic diffuse radiations; 2) the strongest $\mathrm{MeV}$ source (the $\mathrm{Crab}$ ); and 3) neighboring COMPTEL sources on a case by case basis.

To compare our COMPTEL source fluxes with the EGRET ones, we plotted the best-fit power-law shapes to the EGRET source spectra with $1 \sigma$ errors and extrapolated those below $100 \mathrm{MeV}$ into the COMPTEL band. Systematic errors of $10 \%$ are included in these EGRET spectra.
The COMPTEL flux values are given for 4 standard COMPTEL bands $(0.75-1,1-3,3-10,10-30 \mathrm{MeV})$. For a detection significance $<2 \sigma$ the source flux is presented by a $2 \sigma$ upper limit, otherwise by a flux point. Additionally, when comparing with the EGRET spectral extrapolations, the COMPTEL error bars and subsequently also the upper limits are enlarged by 20 percent to account for systematic errors.

\section{Results}

\subsection{General results}

We investigated all unidentified or tentatively identified EGRET sources for which spectral indices are given in the third EGRET catalogue. By excluding 5 artifacts near the Vela pulsar and 1 artifact near Crab (Thompson et al. 2001), an ensemble of 173 sources was selected for our analyses. In some sky regions the unidentified EGRET sources are "crowded". For cases where they are closer than the COMPTEL location accuracy, no precise and unambiguous $\mathrm{MeV}$ flux could be attributed to individual EGRET sources. In these cases only one $\mathrm{MeV}$ source was fitted and its flux value was treated as an upper limit for the different EGRET sources.

In most cases, we derive only upper flux limits or marginal $(<4 \sigma)$ hints of MeV emission. We find significant MeV detections in 4 sky regions; three of them are locations of already known unidentified $\mathrm{MeV}$ sources $((l, b)=(358.5,0.5)$, $(311.5,-2.5),(18.5,-0.5))$. The fourth one, at $\sim(l, b)=(188.7$, -4.4) and dubbed GRO J0550+19 in Bronsveld et al. (2002), is near the location of the Crab, which is by far the strongest $\mathrm{MeV}$ source. Detailed studies of these sky regions have been reported earlier or are in progress (Strong et al. 2001; Zhang et al. 2002; Bronsveld et al. 2002; Collmar et al. 2004).

In general our results allow the sources to be divided into 4 groups. For the majority of sources $(\sim 120)$ COMPTEL cannot provide any constraints on the EGRET spectra (group 1). We detect evidence (detection significance $>2 \sigma$ ) for about 20 sources whose $\mathrm{MeV}$ fluxes are consistent with the EGRET extrapolations, showing that the measured EGRET spectra extend into the MeV band (group 2). A third group contains a few sources indicating a spectral upturn of the EGRET spectra at $\mathrm{MeV}$ energies, suggesting the presence of an additional spectral component. Finally, we found 22 sources whose COMPTEL fluxes or flux upper limits are below the expected fluxes based on extrapolations of their EGRET spectra, requiring a spectral turnover/break at $\mathrm{MeV}$ energies (group 4). In this paper we want to concentrate on the sources of group 4 for which COMPTEL can provide meaningful constraints on the EGRET spectra. Details on the three other groups will be given in a later paper.

\subsection{Sources with spectral constraints in the MeV band}

Applying the method described above, we found a subgroup of 22 unidentified (no tentatively identified source belongs to our sample) EGRET sources for which a spectral flattening at $\mathrm{MeV}$ energies is required. Figure 1 shows four typical examples. All 22 sources are listed in Table 1 including the relevant 

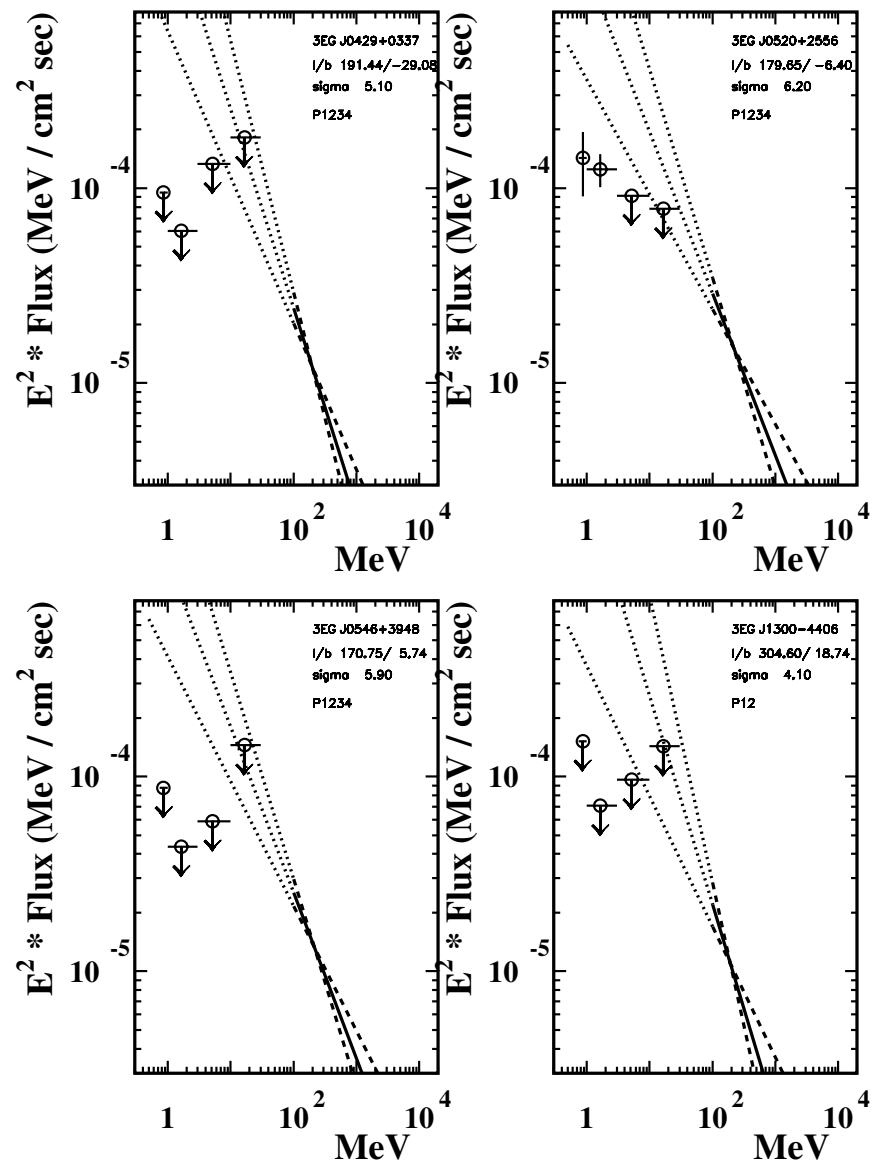

Fig. 1. Combined simultaneous COMPTEL/EGRET energy spectra of 4 sources out of our sample of 22 sources (see Table 1). The inserted text gives the EGRET 3rd catalogue informations (Hartman et al. 1999) on the source (source name, sky location, EGRET detection significance, and detection period). The solid line represents the best-fit EGRET spectrum above $100 \mathrm{MeV}$, the dashed lines its $1 \sigma$ error in spectral index, and the dotted lines the spectral extrapolations below $100 \mathrm{MeV}$ down to $0.75 \mathrm{MeV}$. The required spectral changes at $\mathrm{MeV}$ energies are obvious. The COMPTEL upper limits are $2 \sigma$ and the error bars on the flux points are $1 \sigma$.

source parameters. At least two sources, 3EG J1638-5515 and 3EG J1823-1314, have a $\gamma$-ray luminosity peak in the $1-100 \mathrm{MeV}$ band. Their luminosity or luminosity upper limit in the COMPTEL 1-3 MeV band is significantly lower than measured luminosity values at energies between 10 and $100 \mathrm{MeV}$, i.e. the COMPTEL spectral shape is harder than $E^{-2}$, while the EGRET one is softer than $E^{-2}$. In fact, most likely the majority of sources in this sample has its maximum luminosity in this range, since we know that generally no strong hard X-ray sources have been found in EGRET error boxes.

The spatial distribution of the 22 sources is shown in Fig. 2. A concentration at low galactic latitudes of $|b|<30^{\circ}$ is apparent. The sources tend to concentrate in the inner galactic region. Such a distribution suggests galactic origins for most of these sources.

Figure 3 shows correlation plots of spectral index versus flux at energies above $100 \mathrm{MeV}$ for a) the 22 selected sources and $b)$ the rest $(\sim 151$ sources $)$ of the unidentified and tentatively identified EGRET sources. No obvious correlation between spectral index and flux is visible for both source groups. The linear fit results in average photon indices of $2.72_{-0.11}^{+0.08}$ for the "break" sample and $2.13 \pm 0.03$ for the rest. The softer energy spectra for the "break" sample might be a selection effect due to the worse detection sensitivity of COMPTEL compared to EGRET. For sources having similar flux levels in the EGRET band, those with a softer energy spectrum are more likely to be constrained by the COMPTEL data, because the extrapolation of the EGRET spectrum into the COMPTEL band will reach higher flux values. For the harder sources, the spectral extrapolation might go below the COMPTEL sensitivity limits making COMPTEL constraints impossible.

\subsection{Variability}

There are three approaches to estimate the flux variability of $\gamma$-ray sources. The so-called V method (McLaughlin et al. 1996) is based on the $\chi^{2}$-test. It is affected by the source detection significance, which is in $\gamma$-ray analyses/data often low. For a low-significance source with intrinsic variability a small $V$ value is derived. On the other hand, a large $V$ value can either be due to real source variability or due to large systematic effects. To overcome these systematic problems, Zhang et al. (2000a) and Torres et al. (2001) suggested to use the so-called $I$-index instead. It is defined as the ratio of the measured source variability to those of $\gamma$-ray pulsars, $I=\mu_{\text {source }} / \mu_{\text {pulsars }}$, which are considered to be intrinsically constant. $\mu$ is the ratio of the standard deviation of the measured flux values to the weighted mean flux. We regard a source as variable, if $I$-index $>2.5$ (corresponding to a $3 \sigma$ significance for variability), a source as stable, if $I$-index $<1.5$, and a source as dubious, if its $I$-index value is in between. We calculated the $I$-indices for the 22 sources of our sample according to Torres et al. (2001) by using the flux values given in the 3rd EGRET source catalogue. The results are listed in Table 1 . We found 10 sources to have an $I$-index $>2.5$ (i.e. variable) and two to have an $I$-index $<1.5$ (i.e. constant).

Tompkins (1999) investigated the source variability via the $\tau$-method, which is defined as $\tau=\sigma / \mu$, where $\sigma$ is the standard deviation of the fluxes and $\mu$ the average value. The idea is to overcome systematic effects by comparing to the $\tau$ distribution for sources of known nature. The source fluxes are assumed to have a Gaussian distribution with the parameters $\tau \times \mu$ (width) and $\mu$ (mean value). These parameters are estimated by the maximum likelihood method. By comparing to the $\tau$ distribution of blazars, a source is regarded variable if its $68 \%$ lower limit is greater than 1 . This results in four of our sources being variable. Three of them have an $I$ value $>2.5$ and one, 3EG J1825+2854, has an $I$ value of 2.49. These results show that the $I$ - and $\tau$-methods provide consistent results. All other 18 sources, with $\tau_{\max }$ values larger than 0.3 and $\tau_{\min }$ values less than 1 , would be classified as dubious, according to the definition by Tompkins (1999). Nolan et al. (2003) adopted the same $\tau$-method, only applying slight changes/modifications, to investigate the time 


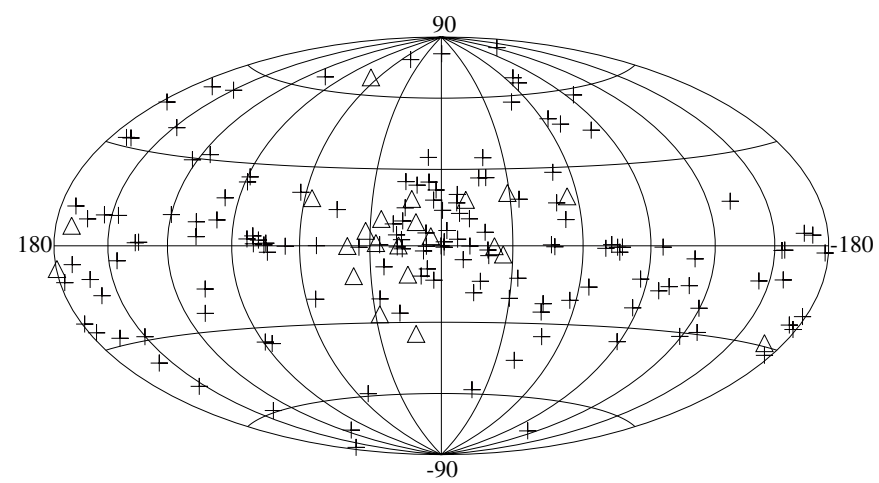

Fig. 2. The sky distribution of 173 unidentified and tentatively identified EGRET sources $(+, \triangle)$ in galactic coordinates. The triangles $(\triangle)$ represent the 22 EGRET sources of our sample. A clustering towards the inner galaxy is indicated. Especially, 20 of the 22 sources are located at $|b|<30^{\circ}$, suggesting a galactic origin.

Table 1. Catalogue of the 22 unidentified EGRET sources which - by inclusion of the COMPTEL results - have to have a spectral break or their $\gamma$-ray luminosity maxima at $\mathrm{MeV}$ energies. The source parameters of the first six columns are taken from the third EGRET catalogue, Cols. 7 and 8 are for the variability analysis, and the last 4 columns give the COMPTEL fluxes/upper limits in the 4 standard energy bands. The abbreviations are the following:

Name - 3EG source name.

l. $b$. - source coordinate of galactic longitude and latitude.

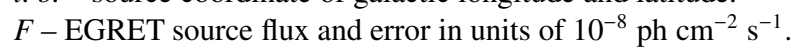

$\alpha$ - photon spectral index and its error.

$V P$ - CGRO observation periods for which the spectral index is derived. P1 means phase 1, P12 the combination of P1 and P2, and so on. Observation 330+ is the combined VPs 330 and 332.

$\tau\left(\tau_{\min }, \tau_{\max }\right)$ - taken from Tompkins (1999) for measuring the fractional variability of EGRET sources. $\tau_{\min }$ and $\tau_{\max }$ represent separately the $68 \%$ lower and upper limits on $\tau$.

$I$ - index defined by Torres et al. (2001) for measuring source variability with respect to $\gamma$-ray pulsars; derived by our variability analysis on the published EGRET fluxes.

Flux $\left(10^{-5} \mathrm{ph} \mathrm{cm}^{-2} \mathrm{~s}^{-1}\right)$ - COMPTEL fluxes and the flux unit in the four standard energy bands: $0.75-1,1-3,3-10$ and $10-30 \mathrm{MeV}$. The upper limits are $2 \sigma$ and the error bars are $1 \sigma$.

\begin{tabular}{|c|c|c|c|c|c|c|c|c|c|c|c|}
\hline \multirow{2}{*}{$\begin{array}{l}\text { Name } \\
\text { 3EG J }\end{array}$} & \multirow{2}{*}{$\begin{array}{c}l \\
\operatorname{deg}\end{array}$} & \multirow{2}{*}{$\begin{array}{c}b \\
\operatorname{deg}\end{array}$} & \multirow[t]{2}{*}{$F$} & \multirow[t]{2}{*}{$\alpha$} & \multirow[t]{2}{*}{$\overline{\mathrm{VP}}$} & \multirow[t]{2}{*}{$\tau\left(\tau_{\min }, \tau_{\max }\right)$} & \multirow[t]{2}{*}{$I$} & \multicolumn{4}{|c|}{ Flux $\left(10^{-5} \mathrm{ph} \mathrm{cm}^{-2} \mathrm{~s}^{-1}\right)$} \\
\hline & & & & & & & & $0.75-1$ & $1-3$ & $3-10$ & $10-30$ \\
\hline $0429+0337$ & 191.44 & -29.08 & $2.0 \pm 2.7$ & $2 \pm 0.27$ & 4 & $00,0.45)$ & 1.6 & $<3.2$ & $<4.0$ & $<3.1$ & $<1.2$ \\
\hline $0520+2556$ & 179.65 & -6.40 & $15.7 \pm 2.7$ & $2.83 \pm 0.24$ & $\mathrm{P} 1234$ & $0.00(0.00,0.31)$ & 1.1 & $4.8 \pm 1.7$ & $8.3 \pm 1.6$ & $<2.1$ & $<0.5$ \\
\hline $0546+3948$ & 170.75 & 5.74 & $13.7 \pm 2.6$ & $2.85 \pm 0.21$ & $\mathrm{P} 1234$ & $0.11(0.00,0.47)$ & 1.7 & $<2.9$ & $<2.9$ & $<1.4$ & $<0.9$ \\
\hline $1300-4406$ & 304.6 & 18.74 & $10.6 \pm 2.9$ & $3.07 \pm 0.40$ & $\mathrm{P} 12$ & $0.48(0.00,1.57)$ & 3.0 & $<5.0$ & $<4.7$ & $<2.2$ & $<1.0$ \\
\hline $1424+3734$ & 66.82 & 67.76 & $16.3 \pm 4.9$ & $3.25 \pm 0.46$ & $\mathrm{P} 1$ & $0.01(0.00, \infty)$ & 1.9 & $<6.4$ & $8.5 \pm 3.1$ & $<5.6$ & $<2.2$ \\
\hline $1500-3509$ & 330.91 & 20.45 & $10.9 \pm 2.8$ & $2.99 \pm 0.37$ & $\mathrm{P} 1234$ & $0.00(0.00,0.61)$ & 1.5 & $<5.1$ & $<3.3$ & $<1.6$ & $<1.0$ \\
\hline $1612-2618$ & 349.40 & 17.90 & $92.2 \pm 27.7$ & $2.71 \pm 0.23$ & 423. & $1.78(0.76$ & 4.1 & $<41.0$ & $<1$ & $<9.3$ & $<4.2$ \\
\hline $1638-5515$ & 334.05 & -3.34 & $67.3 \pm 14.2$ & $2.56 \pm 0.21$ & $\mathrm{P} 2$ & $0.00(0.00,0.69)$ & 2.4 & $<15.6$ & $<7.0$ & $<6.7$ & $2.8 \pm 1.0$ \\
\hline $1639-4702$ & 337.75 & -0.15 & $53.2 \pm 8.7$ & $2.50 \pm 0.18$ & $\mathrm{P} 1234$ & $0.00(0.00,0.38)$ & 2.0 & $<3.1$ & $<4.5$ & $<3.0$ & $<0.9$ \\
\hline $1709-0828$ & 12.86 & 18.25 & $12.6 \pm 3.2$ & $3.00 \pm 0.35$ & $\mathrm{P} 1234$ & $0.84(0.11,2.21)$ & 2.7 & $<3.2$ & $<3.2$ & $<2.8$ & $<0.6$ \\
\hline $1735-1500$ & 10.73 & 9.22 & $196.3 \pm 48.8$ & $3.24 \pm 0.47$ & 231.0 & $1.09(0.00,10.14)$ & 8.9 & $<29.0$ & $<23.1$ & $<11.8$ & $<4.5$ \\
\hline $1741-2312$ & 4.42 & 3.76 & $33.1 \pm 5.9$ & $2.49 \pm 0.14$ & $\mathrm{P} 12$ & $0.52(0.18,1.03)$ & 2.2 & $<11.9$ & $<4.4$ & $<3.0$ & $<0.9$ \\
\hline $1800-0146$ & 25.49 & 10.39 & $26.1 \pm 6.1$ & $2.79 \pm 0.22$ & P34 & $0.00(0.00,0.48)$ & 1.9 & $<8.1$ & $<7.0$ & $<2.2$ & $<1.4$ \\
\hline $1823-1314$ & 17.94 & 0.14 & $102.6 \pm 12.5$ & $2.69 \pm 0.19$ & P3 & $0.72(0.40,1.37)$ & 3.0 & $10.2 \pm 2.9$ & $5.6 \pm 2.8$ & $3.7 \pm 1.2$ & $2.7 \pm 0.5$ \\
\hline $1825+2854$ & 56.79 & 18.03 & $34.3 \pm 10.9$ & $4.47 \pm 1.15$ & 9.2 & $73.19(2.59, \infty)$ & 2.5 & $<30.1$ & $<13.9$ & $<8.6$ & $<2.2$ \\
\hline $1828+0142$ & 31.90 & 5.78 & $132.2 \pm 24.0$ & $2.76 \pm 0.39$ & 13.1 & $3982.15(6.92, \infty)$ & 5.3 & $<16.4$ & $<15.8$ & $<11.3$ & $<4.4$ \\
\hline $1837-0423$ & 27.44 & 1.06 & $310.4 \pm 63.7$ & $2.71 \pm 0.44$ & 423.0 & $12.01(2.17, \infty)$ & 8.4 & $<23.4$ & $<18.5$ & $<14.3$ & $<3.0$ \\
\hline $1858-2137$ & 14.21 & -11.15 & $11.2 \pm 2.6$ & $3.45 \pm 0.38$ & $\mathrm{P} 1234$ & $0.00(0.00,0.56)$ & 2.8 & $<4.2$ & $<5.0$ & $1.6 \pm 0.7$ & $<0.6$ \\
\hline $1903+0550$ & 39.52 & -0.05 & $62.1 \pm 8.9$ & $2.38 \pm 0.17$ & $\mathrm{P} 1234$ & $0.35(0.18,0.60)$ & 2.3 & $<3.3$ & $<3.4$ & $<2.7$ & $<0.7$ \\
\hline $1940-0121$ & 37.41 & -11.62 & $41.0 \pm 10.7$ & $3.15 \pm 0.39$ & $330 .+$ & $4.58(1.13, \infty)$ & 4.0 & $<16.6$ & $<10.9$ & $<6.8$ & $<1.8$ \\
\hline $2020-1545$ & 28.09 & -26.62 & $11.8 \pm 3.4$ & $3.40 \pm 0.55$ & P1 & $0.00(0.00,0.80)$ & 0.9 & $<5.2$ & $<7.7$ & $<2.6$ & $<1.0$ \\
\hline 2034-3110 & 12.25 & -34.64 & $17.4 \pm 5.2$ & $3.43 \pm 0.78$ & P1 & $2.88(0.89,154.84)$ & 5.7 & $<5.4$ & $<5.4$ & $<3.2$ & $<1.7$ \\
\hline
\end{tabular}



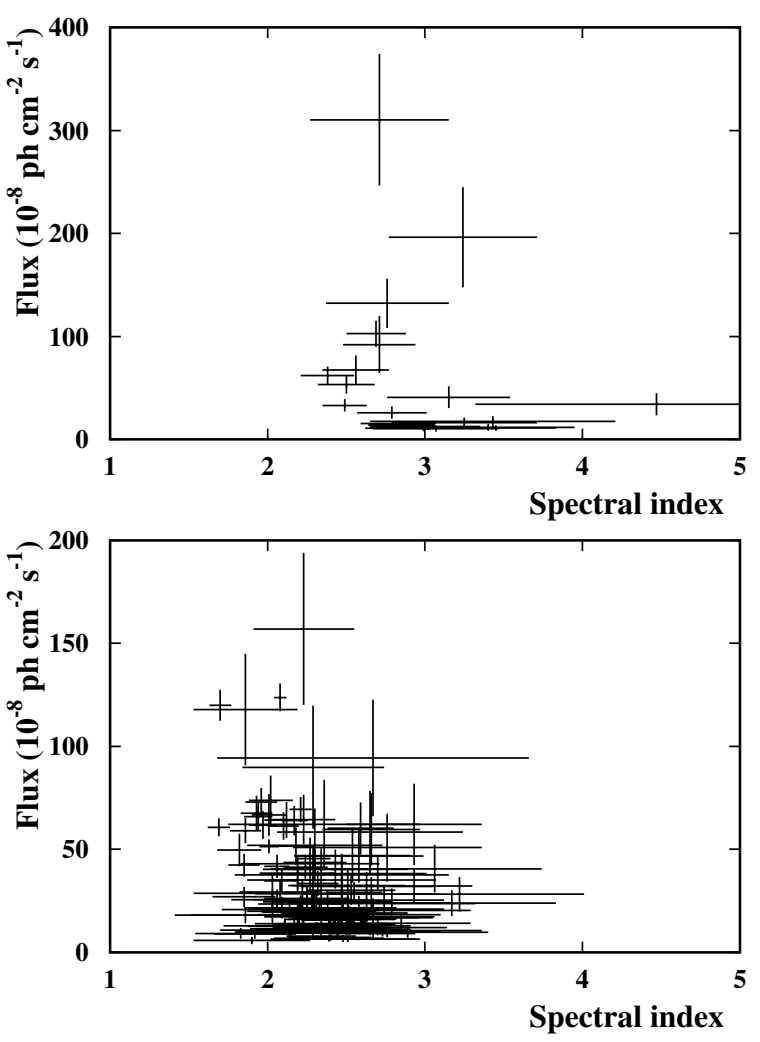

Fig. 3. Correlation plots of spectral photon index versus flux (EGRET results) for our sample of 22 sources (upper panel) and the rest of 151 unidentified and tentatively identified EGRET sources (lower panel). The sources of our sample typically have softer spectra.

variability of the EGRET $\gamma$-ray sources. Their approach resulted in some numerical differences to Tompkins. According to the classification by Nolan et al. (2003), four sources of our Table 1, 3EG J1612-2618,1828+0142, 1837-0423, 1940-0121 and 2034-3110, are variable. These sources have $I$-indices $>$ 2.5. All others, with the exception of 3EG J1424+3734, which is not included in Nolan et al. (2003) due to their selection criteria on the observations, are classified as dubious. Since the $\tau$ values are estimated by lumping the VPs within one month while the $I$-index method uses individual VPs, typically ranging from several days to two weeks, the two methods may estimate the source variability on different time scales. We use the $I$-index to classify the source variability. This yields, as mentioned above, 10 variable sources. Because of the large $I$-index value (2.49) and the variability-indicating $\tau$ value

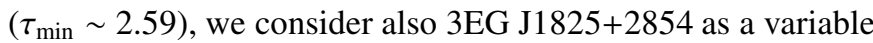
source. The two sources with $I$-index $<1.5$ are considered dubious due to their $\tau$ values. Thus we have a sample of 11 variable sources and of 11 dubious sources.

Figure 4 shows correlation plots of spectral photon index versus $I$-index for the 22 sources of our sample and the remaining 151 sources. For the sample of the 151 sources, the linear correlation coefficient is derived to be $\sim 0.15$, suggesting that the softer sources tend to be more variable. This is consistent with Torres et al. (2001), who found that the mostvariable unidentified EGRET sources near the galactic plane
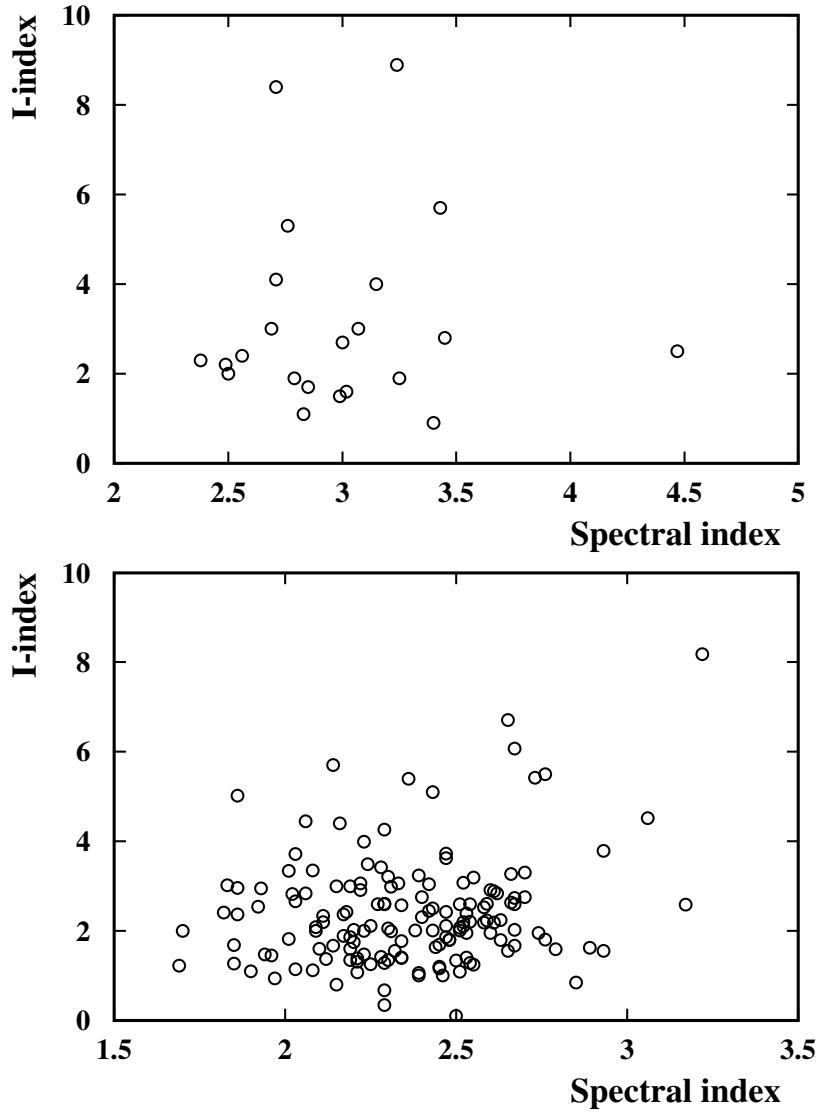

Fig. 4. Correlation plots of spectral photon index versus variability $I$-index for our sample of 22 sources (upper panel) and the rest of 151 unidentified and tentatively identified EGRET sources (lower panel). The lower frame indicates a trend for higher variability with softer spectra.

tend to have steep spectra. The 22 sources do not show such a trend. A linear correlation coefficient of $\sim 0.03$ indicates an uncorrelated sample. This might either discriminate this sample of 22 sources from the rest, or is due to the relatively poor statistics.

\section{Discussion}

By analyzing the COMPTEL data of unidentified and tentatively identified EGRET sources, and comparing their COMPTEL spectra with those from EGRET, we found a subgroup of 22 sources which show a spectral turnover/break at $\mathrm{MeV}$ energies. At least two of them, but most likely the majority, have an emission maximum at energies between 1-100 MeV. Variability analyses show that half of the sources are variable above $100 \mathrm{MeV}$. For the other half no significant time variability could be proven.

The properties of the variable sources are reminiscent of the observational properties of EGRET-detected blazars. They are generally variable and their broadband (radio to $\gamma$-ray) spectra are characterized by two spectral maxima. The high-energy one is for many sources located at MeV energies, e.g. 3C 273 (Lichti et al. 1995), PKS 0528+134 (Collmar et al. 1997; 
Mukherjee et al. 1999), 3C 279 (Hartman et al. 2001). For details on their high-energy emission see e.g. Collmar (2001) and references therein. Because the EGRET blazars are typically high-latitude sources, variable unidentified high-latitude EGRET sources are generally considered to be of extra-galactic blazar origin. Due to EGRET's decreasing sensitivity to variability with decreasing flux they can appear as non-variable sources at low latitudes (McLaughlin et al. 1996), where, due to the strong diffuse galactic emission, a higher significance level for source detection is required. Therefore some of the lowlatitude unidentified EGRET sources may also be blazars. Two sources of our sample, 3EG J1424+3734 and 3EG J2034-3110, are high-latitude $\left(|b|>30^{\circ}\right)$ sources. 3EG J2034-3110 is variable and 3EG J1424+3734 is dubiously variable. Our finding of a "blazar-like" $\mathrm{MeV}$ spectrum provides further evidence for the blazar nature of these 2 sources, especially for the case of 3EG J2034-3110, for which time-variability is observed. The lack of an obvious blazar candidate at these source positions can be explained by the calculations of Torres et al. (2002). The unidentified $\gamma$-ray sources could be a distant weak $\gamma$-ray emitting blazars whose emission is amplified by gravitational microlensing. The magnification factor is energy dependent and a broken energy spectrum is predicted. Although the individual source properties of our sample sources are reminiscent of blazars, their distribution on the sky is not. While the blazars are mainly detected at high latitudes, our source group concentrates towards the inner galaxy. Therefore we conclude that at least the majority of these sources are not of blazar origin.

Some $\gamma$-ray pulsars also show such an MeV-turnover spectrum. One example is PSR B1509-58. Kuiper et al. (1999) showed that its maximal luminosity is reached between 10 and $30 \mathrm{MeV}$, followed by a strong softening of its spectrum towards the EGRET band. In fact, PSR B1509-58 has not been detected by EGRET above $100 \mathrm{MeV}$, while it is a strong COMPTEL source. PSR 1509-58 is a young pulsar and its surface magnetic field can be inferred as at least $3.1 \times 10^{13} \mathrm{G}$ (Kuiper et al. 1999). In such sources, electrons are accelerated up to relativistic energies, and subsequently produce $\gamma$-rays via curvature radiation and inverse-Comptonization of soft photons. In the vicinity of the magnetic pole, $\gamma$-rays can be absorbed by the strong magnetic field via the photon splitting process. The latter process happens if the magnetic field is larger than a critical value $B>0.3 B_{\mathrm{cr}}$, where $B_{\mathrm{cr}}=4.413 \times$ $10^{13} \mathrm{G}$ is the surface magnetic field (Harding et al. 1997). Its onset has no energy threshold. For such a strong magnetic field, the polar cap scenario predicts that photon splitting can become the dominant attenuation process, resulting in a softening of the energy spectrum below $100 \mathrm{MeV}$, i.e. providing the observed $\mathrm{MeV}$ cutoff. It should be mentioned that also in the competing outer gap scenario such a spectral break at low $\gamma$-ray energies can be accounted for. Namely, Zhang \& Cheng (2000b) applied their three dimensional outer magnetosphere model to PSR B1509-58. They could reproduce the measured broad pulse profile and the measured pulsed energy spectrum from the optical range up to $\gamma$-rays. This spectrum shows a power-law shape with a spectral bend above $1 \mathrm{MeV}$. In the same work, Zhang \& Cheng considered also the case of the young Crab-like LMC pulsar PSR B0540-69, which is also not detected by EGRET but below $10 \mathrm{keV}$ stronger than PSR B1509-58. The overall characteristics of these two young pulsars appear rather similar. By analyzing RXTE data, De Plaa et al. (2003) showed that PSR B0540-69 and the Crab pulsar have very similar spectral shapes up to about $50 \mathrm{keV}$, where the Crab pulsar spectrum reaches its maximum luminosity. From these hard X-rays to the EGRET energies above $100 \mathrm{MeV}$, the Crab spectrum softens throughout the COMPTEL range (photon index -2.4; Kuiper et al. 2001). De Plaa et al. discussed the spectral shapes of these three very young pulsars $\left(\leq 1.6 \times 10^{3} \mathrm{yr}\right)$ which appear to be different from those of older $\gamma$-ray pulsars like Vela and Geminga. The youngest pulsars are strong(er) in the X-ray domain, but weak(er) above $100 \mathrm{MeV}$. Such a spectral behaviour makes them less likely counterparts to the unidentified EGRET sources, because their $L_{\mathrm{X}} / L_{\gamma}$ ratio is too high. On the other hand, all older established $\gamma$-ray pulsars like Vela, PSR B1706-44, PSR B1951+32, Geminga and PSR B1055-52 are weak X-ray emitters but have harder $\gamma$-ray spectra extending up to the $\mathrm{GeV}$ range. The latter might be a selection effect. For example, the weakly detected (above $50 \mathrm{MeV}$ ) $\gamma$-ray pulsar PSR B0656+14 exhibits a steep spectrum (index $=-2.8 \pm 0.3$ ) at energies above $100 \mathrm{MeV}$ (Ramanamurthy et al. 1996). This pulsar has a modest surface magnetic field of $4.7 \times 10^{12} \mathrm{G}$ and a characteristic age of $1.1 \times 10^{5}$ years. It has not been detected by COMPTEL below $30 \mathrm{MeV}$, implying a turnover of the soft EGRET spectrum before the COMPTEL window, just like our sample.

Another pulsar type, the old, recycled weakly magnetized millisecond pulsars, can also show such an MeV-turnover spectrum. Namely, a $4.9 \sigma$ detection has been claimed of the 2.3 ms pulsar PSR J0218+4232 (Kuiper et al. 2000, 2002) at EGRET energies. Its spin down parameters give a rather old characteristic age of $4.6 \times 10^{8}$ years. Its magnetic field of $4.3 \times$ $10^{8} \mathrm{G}$ is much weaker than that of standard $\gamma$-ray pulsars (e.g. Crab, Geminga). A weak, but very hard X-ray spectrum was measured (photon index below $10 \mathrm{keV}>-1$; Mineo et al. 2000) and a rather soft $\gamma$-ray spectrum (photon index $\sim-2.6$ ) was detected for EGRET above $100 \mathrm{MeV}$. Current polar cap and outer gap models can account for the production of such high-energy radiation in millisecond pulsars, but have difficulties in reproducing the observed spectral shape. Nevertheless, the observed spectrum has the overall spectral shape of our source sample, making millisecond pulsars viable candidate counterparts, but only for the stable EGRET sources. Millisecond pulsars are expected to be stable high-energy emitters just like the normal radio pulsars, and contrary to the case of accreting pulsars for which variability is naturally expected.

$\mathrm{X}$-ray binaries (XRBs) have also been suggested as counterparts of unidentified EGRET sources. One XRB, the neutron star system Cen X-3, has been detected as $\gamma$-ray emitter during an activity period in 1994 (Vestrand et al. 1997). EGRET found a temporary $5 \sigma$ source which was positionally coincident with Cen X-3. The $4.8 \mathrm{~s}$ modulation of the $\gamma$-rays, coinciding with the $4.8 \mathrm{~s}$ rotation period of the neutron star, provided compelling evidence for the identification (Vestrand et al. 1997). A hard power-law spectrum (photon index $-1.81 \pm 0.37$ ) was measured between $70 \mathrm{MeV}$ and $10 \mathrm{GeV}$. The authors suggest that galactic X-ray binary 
systems may constitute a class of highly variable $\mathrm{GeV} \gamma$-ray sources. It is assumed that in Cen X-3 we see the unabsorbed $\gamma$-ray spectrum. If in such a system the radiation region is surrounded by a condensed soft photon field, e.g. in an accreting $\mathrm{XRB}$, an energy-dependent absorption of the $\gamma$-rays will occur resulting in a soft $\gamma$-ray spectrum. This scenario was proposed by Romero et al. (2001) for the possible association of the EGRET source 3EG J0542+2610 and the Be/X-ray transient $\mathrm{A} 0535+26$. Such an absorption process was also studied by Wu et al. (1993) for the XRB Cyg X-3. Their simulations showed that the $\gamma$-ray spectrum can change significantly when passing through the ambient soft X-ray field of an accreting source. The $100 \mathrm{MeV}-1 \mathrm{GeV}$ emission will be absorbed. For both cases a soft $\gamma$-ray spectrum would be observable by EGRET, if the absorption is not too strong.

The emission processes of microquasars/blazars and extragalactic blazars are - in principle - the same, however, on different time, space, and energy scales. Therefore one expects microquasars as potential counterparts of the unidentified EGRET sources. Paredes et al. (2000) suggest the microquasar LS 5039 to be the counterpart of 3EG J1824-1514. Calculations of microquasar spectra show that, depending on the strength of the jet Lorentz factor and magnetic field, microquasars could be detected by EGRET, and that they could have their spectrum turnover at $\mathrm{MeV}$ energies (e.g., Kaufman Bernado et al. 2002). However, no microquasar was definitely identified yet as an EGRET source. Given the current knowledge, the properties of some of our sources, spectral turnover and variability, could be matched by microquasars.

Binaries composed of early-type stars, like Wolf Rayet, Of and Be stars, which produce strong stellar winds are proposed by Benaglia \& Romero (2003) to be potential $\gamma$-ray emitters. They argued that the electrons could be accelerated to relativistic energies by the shocks generated in the colliding wind region of the early-type binaries, and then cool via the process of inverse-Compton scattering off the local soft photon field. A low-energy cut off of the electron spectrum or incomplete cooling of the electron population could lead to a turn over in the $\gamma$-ray spectrum at lower $\gamma$-ray energies. The early-type binaries are concentrated in the inner spiral arms of the Galaxy and variability is expected due to the changing geometry. However, according to the investigation of Romero et al. (1999) on the spatial correlation of low-latitude unidentified EGRET sources with early-type stars, none of the 22 sources of our sample is spatially coincident with any early-type binary.

Another class of sources proposed to be candidates for unidentified EGRET sources are supernova remnants (SNRs) (e.g., Esposito et al. 1996). In SNRs electrons and/or protons can be accelerated by the Fermi process to relativistic energies or appear in the outflows of a pulsar, if one is embedded in the SNR. Subsequently $\gamma$-rays can be produced via processes of inverse-Compton scattering, relativistic bremsstrahlung, synchrotron emission, or $\pi_{0}$ decay. Since the first-order Fermi process has a monotonic evolution of energies with time and the acceleration time for the maximum energy is limited by the age of the remnant, it is generally thought that SNRs should be stable $\gamma$-ray sources, at least on a time scale of several years. SNRs are spatially large, have ambient surrounding matter and a relatively weak magnetic field. A spectral turnover at MeV energies as found for our sample could be generated by inverseComptonization of soft photons, by having the maximum of the synchrotron emission at $\mathrm{MeV}$ energies (e.g. Crab nebula), or by the decay of $\pi_{0}$ particles (generated in p-p collisions). The latter one would result in a broad spectral bump centered around $68 \mathrm{MeV}$. Four sources of our sample, 3EG J1639$4702,1823-1314,1837-0423,1903+0550$, are positionally coincident with SNRs. 3EG J1823-1314 and 3EG J1837-0423 are variable (Table 1) and therefore are unlikely counterparts of the SNRs. For 3EG J1639-4702 and 3EG J1903+0550 no variability is proven, therefore they remain potential counterparts for the EGRET $\gamma$-ray sources. Their possible associations with SNRs and coinciding radio pulsars have been discussed by Torres et al. (2003). With respect to radio pulsars, they concluded based on the energetics, that for 3EG J16394702 only one of the coinciding radio pulsar, PSR J1637-4642, has the potential to be the counterpart. For 3EG J1903+055 they did not find a potential pulsar counterpart, although they note that one of the coinciding pulsar lacks information and therefore can not be judged. With respect to $\gamma$-ray emission due to $\pi_{0}$-decay, Torres et al. point out that the SNRs are too far away to generate the observed flux. Even considering an enhancement of the $\gamma$-ray production due to interactions of the accelerated nuclei with nearby dense molecular clouds, they find the identifications to be unlikely. These two unidentfied sources could be associated with a pulsar wind nebula, analogous to the case of the Crab for which the electrons might be accelerated in the inner nebula and subsequently are generating a synchrotron spectrum which cuts off at $\mathrm{MeV}$ energies (de Jager et al. 1996). Their fluxes above $100 \mathrm{MeV}$ have about the level of the Crab nebula. Because with distances of at least $8 \mathrm{kpc}$ (Torres et al. 2003), i.e. at least 4 times the distance to the Crab, they would have to be significantly more powerful at EGRET energies than the Crab nebula. However, this could be possible if their synchrotron cut off would be shifted to higher energies (higher characteristic synchrotron energy) compared to the Crab nebula.

Because for many unidentified EGRET sources no obvious candidate counterpart exists, Romero et al. (1999) mentioned isolated rotating black holes, standard (Kerr) or charged (Kerr-Newman) ones, as possible counterparts. They could accrete from the diffuse interstellar medium (ISM). Changes in the density of the ISM would result in a variable $\gamma$-ray flux. One such source, 3EG J1828+0142, was modeled by Punsly et al. (2000) by assuming an isolated Kerr-Newman black hole origin. The model predicts a steep synchrotron selfCompton spectrum with a spectral maximum at $\mathrm{MeV}$ energies, i.e. matching the spectral properties of our source sample. Including 3EG J1828+0142, seven of our sample sources (3EG J0520+2556, 0546+3948, 1638-5515, 1735-1500, 1741$2312,1800-0146,1828+0142)$ are located at low galactic latitudes $\left(|b|<10^{\circ}\right)$ and lack any positional coincidence with galactic objects of potential counterpart nature. Two of them, 3EG J1735-1500 and 3EG 1828+0142, are significantly variable and therefore match the anticipated $\gamma$-ray properties of these exotic objects. 


\section{Conclusion}

By analyzing the contemporary COMPTEL observations of all unidentified or only tentatively identified EGRET sources, we found a subgroup of 22 sources for which we can provide spectral constraints for source modelling. Their spectra have to turn over between $\sim 1 \mathrm{MeV}$ and $100 \mathrm{MeV}$, and at least two of them, but most likely the majority, have their maximum luminosities somewhere in this energy band. Most of the sources are not detected by COMPTEL, however the simultaneously derived upper limits require the spectral bending. These sources have rather steep energy spectra in the EGRET band, and seem to be preferentially located in the inner galaxy, especially at low latitudes $\left(|b|<30^{\circ}\right)$. Variability studies reveal that half of them are significantly variable above $100 \mathrm{MeV}$. Potential counterparts have to conform to these observational results. A blazar origin for the two high-latitude sources in this sample seems to be likely. Viable candidate counterparts for the steady low-latitude sources are: 1) young (age $<10^{6}$ years) pulsars, although the youngest with the strongest magnetic fields might be too strong in the X-ray domain; 2) old, recycled millisecond pulsars with a weak magnetic field (like PSR J0218+4232); and 3) SNRs and pulsar wind nebula whose synchrotron spectra are peaking at $\mathrm{MeV}$ energies (like the Crab nebula). For the variable low-latitude sources, XRBs, in particular microquasars/blazars by assuming a spectral analogy to the extragalactic objects, and isolated BHs would match the requirements. Case by case studies might reveal further insights in the nature of individual sources.

Acknowledgements. This research was supported by the German government through DLR grant 50 QV 9096 8, by NASA under contract NAS5-26645, and by the Netherlands Organization for Scientific Research NWO. S. Zhang was also subsidized by the Special Funds for Major State Basic Research Projects and by the National Natural Science Foundation of China under grant 10373013.

\section{References}

Benaglia, P., \& Romero, G. E. 2003, A\&A, 399, 1121

de Boer, H., Bennett, K., Bloemen, H., et al. 1992, in Data Analysis in Astronomy IV, ed. V. Di Gesù, L. Scarsi, \& R. Buccheri et al. (New York: Plenum Press), 241

Bloemen, H., Hermsen, W., Swanenburg, B. N., et al. 1994, ApJS, 92, 419

Bronsveld, C. P., Hermsen, W., Kuiper, L., \& Schönfelder, V. 2002, in The Gamma-Ray Universe (Proceedings of the XXXVIIth Rencontres de Moriond), ed. A. Goldwurm, D. N. Neumann, \& J. T. T. Vân (Vietnm: Thẽ Giòi Publishers), 113

Collmar, W., Bennett K., Bloemen, H., et al. 1997, A\&A, 328, 33
Collmar., W. 2001, in Exploring the Gamma-Ray Universe, Proc. of the Fourth INTEGRAL Workshop, ed. A. Giminez, V. Reglero, \& C. Winkler (Noordwijk: ESA Publications Division), 241

Collmar., W., et al. 2004, A\&A, in preparation

De Plaa, J., Kuiper, L., \& Hermsen, W. 2003, A\&A, 400, 1013

Esposito, J. A., Hunter, S. D., Kanbach, G., \& Sreekumar, P. 1996, ApJ, 461, 820

Gehrels, N., Macomb, D. J., Bertsch, D. L., Thompson, D. J., \& Hartman, R. C. 2000, Nature, 404, 363

Harding, A., Baring, M., \& Gonthier, P. 1997, ApJ, 476, 246

Hartman, R. C., Bertsch, D. L., Bloom, S. D., et al. 1999, ApJS, 123, 79

Hartman, R. C., Böttcher, M., Aldering, G., et al. 2001, ApJ, 553, 683 de Jager, O. C., Harding, A. K., Michelson, P. F., et al. 1996, ApJ, 457, 253

Kaufman Bernadó, M. M., Romero, G. E., \& Mirabel, I. F. 2002, A\&A, 355, L10

Kuiper, L., Hermsen, W., Krijger, J. M., et al. 1999, A\&A, 351, 119

Kuiper, L., Hermsen, W., Verbunt, F., et al. 2000, A\&A, 359, 615

Kuiper, L., Hermsen, W., Cusumano, G., et al. 2001, A\&A, 378, 918

Kuiper, L., Hermsen, W., Verbunt, F., et al. 2002, ApJ, 577, 917

Lichti, G. G., Balonek, T., Courvoisier, T. J.-L., et al. 1995, A\&A, 298, 711

McLaughlin, M. A., Mattox, J. R., Cordes, J. M., \& Thompson, D. J. 1996, ApJ, 473, 763

Mineo, T., Cusumano, G., Kuiper, L., et al. 2000, A\&A, 355, 1053

Mukherjee, R., Böttcher, M., Hartman, R. C., et al. 1999, ApJ, 527, 132

Nolan, P. L., Tompkins, W. F., Grenier, I. A., et al. 2003, ApJ, 597, 615

Paredes, J. M., Marti, J., Ribó, M., \& Massi, M. 2000, Science, 288, 2340

Punsly, B., Romero, G. E., Torres, D. F., \& Combi, J. A. 2000, A\&A, 364, 552

Ramanamurthy, P. V., Fichtel, C. E., Kniffen, D. A., Sreekumar, P., \& Thompson, D. J. 1996, ApJ, 458, 755

Romero, G. E., Benaglia, P., \& Torres, D. F. 1999, A\&A, 348, 868

Romero, G. E., Kaufman Bernadó, P., Combi, J. A., \& Torres, D. F. 2001, A\&A, 376, 599

Schönfelder, V., Aarts, H., Bennett, K., et al. 1993, ApJS, 86, 657

Schönfelder, V., Bennett, K., Blom, J. J., et al. 2000, A\&AS, 143, 145

Strong, A. W., Collmar, W., Bennett, K., et al. 2001, in Proc. of Gamma-Ray Astrophysics 2001, AIP Conf. Proc., 587, 21

Tompkins, W. 1999, Ph.D. Thesis, Standford University

Thompson, D. J., Bertsch, D. L., \& Hartman, R. C. 2001, in Proc. of Gamma-Ray Astrophysics 2001, AIP Conf. Proc., 587, 668

Torres, D. F., Romero, G. E., Combi, J. A., et al. 2001, A\&A, 370, 468

Torres, D. F., Romero, G. E., \& Eiroa, E. F. 2002, ApJ, 569, 600

Torres, D. F., Romero, G. E., Dame, T. M., et al. 2003, Phys. Rep., 382,303

Vestrand, W. T., Sreekumar, P., \& Mori, M. 1997, ApJ, 483, L49

Wu, M., Zhang, C. S., \& Li, T. P. 1993, Ap\&SS, 204, 141

Zhang, S., Collmar, W., \& Schönfelder, V. 2002, A\&A, 396, 923

Zhang, L., Zhang, Y. J., \& Cheng, K. S. 2000a, A\&A, 357, 957

Zhang, L., \& Cheng, K. S. 2000b, A\&A, 363, 575 